\title{
Calcium Deficient Hydroxyapatite for Medical Application Prepared by Hydrothermal Method
}

\author{
Koji Ioku ${ }^{{ }^{*}}$, Masanobu Kamitakahara ${ }^{1}$ and Tohru Ikeda ${ }^{2}$ \\ ${ }^{1}$ Graduate School of Environmental Studies, Tohoku University, Aoba 6-6-20 Aramaki, Aoba-ku, \\ Sendai 980-8579, Japan*ioku@mail.kankyo.tohoku.ac.jp, +81-22-795-7407 \\ ${ }^{2}$ Graduate School of Biomedical Sciences, Nagasaki University, 1-7-1 Sakamoto, \\ Nagasaki 852-8588, Japan
}

\begin{abstract}
Hydrothermal processing plays a key role in the synthesis of biomaterials with excellent biocompatibility in the physiological environment. Especially, calcium phosphates are paid to much attention for the regenerative medicine. Two kinds of porous materials of hydroxyapatite with $70 \%$ porosity were prepared. One of them is a newly developed calcium-deficient hydroxyapatite composed of rod-shaped particles of about $20 \mu \mathrm{m}$ in length synthesized hydrothermally (HHA) and the other one is the stoichiometric hydroxyapatite (SHA) synthesized by the conventional sintering method. These materials were used for animal implantation tests to compare these biological responses. In the rabbit femur, implanted HHA was slowly resorbed and then most of the implanted HHA was resorbed after 72 weeks. The implanted SHA was unresorbed throughout the experimental period. The volume of newly formed bone and the number of osteoclasts in the implanted region were significantly larger in HHA than in SHA after 24 weeks. Results in the present research suggested that the activity of osteoclasts correlated to the bone forming activity of osteoblasts. The method to synthesize biodegradable pure calcium-deficient HA is expected to provide adequate biodegradability and bone replaceability.
\end{abstract}

Keywords: Hydroxyapatite Calcium Phosphate, Hydrothermal PACS: 87.85.J

\section{INTRODUCTION}

Hydroxyapatite (HA) is an acid-degradable material, but stoichiometric $\mathrm{HA}\left(\mathrm{Ca}_{10}\left(\mathrm{PO}_{4}\right)_{6}(\mathrm{OH})_{2}\right)$ is basically nonbiodegradable when used as a bone substitute [1, 2]. Osteoclastic bone resorption occurs in conjunction with acidity and proteases supplied from the ruffled border of active osteoclasts. The reason why stoichiometric HA (SHA) is non-biodegradable is a very important question in the field of biomaterial science, but the answer is not clear. It is very difficult to synthesize pure calcium-deficient HA, and difficulty in comparing the biodegradability to that of pure SHA is one reason why we are unable to answer the question. Previously, the authors applied a hydrothermal method to synthesize $\mathrm{HA}$ and beta tricalcium phosphate ( $\beta$-TCP) $[3,4]$. In the hydrothermal method, the suitable conditions enabled the synthesis of HA composed of hexagonal crystals with a prolonged $c$-axis. Hydrothermally synthesized HA with prolonged $c$-axis hexagonal crystals revealed rod-shaped particles by scanning electron microscopy (SEM), and X-ray diffractometry (XRD) indicated that the material was uniform calcium-deficient HA $[5,6]$. Using hydrothermally synthesized HA composed of rod-shaped particles (HHA), it may be possible to clarify the influence of calcium deficiency and microstructure of HA on bioreactivity. In this study, HHA and SHA were implanted in rabbit femurs and histologically analyzed the biological responses.

\section{MATERIALS AND METHODS}

\section{Preparation and Characterization of Materials}

Cylindrical implants of HHA, (6 mm $\phi$ X $10 \mathrm{mmL}$ ) were prepared using an applied hydrothermal method described previously [7]. The $\alpha$-tricalcium phosphate $(\alpha$-TCP) powder was formed into a cylindrical shape by uniaxial compressing. The formed sample was set in a $105 \mathrm{~cm}^{3}$ autoclave with $10 \mathrm{~cm}^{3}$ of pure water and exposed to solution vapor at $200{ }^{\circ} \mathrm{C}$ under saturated vapor pressure for $5 \mathrm{~h}$ (Fig. 1). SHA implants of the same cylindrical shape and size were prepared from stoichiometric HA powder by normal sintering at 1100 ${ }^{\circ} \mathrm{C}$ in air. For each implant, holes of $350 \mu \mathrm{m}$ in diameter were created vertically and horizontally. Samples were characterized by chemical analysis by ICP-MS (Seiko Inst., SPQ9000S, Japan) and XRD (RINT-2100VL, Rigaku, Japan). The surface of samples was analyzed by SEM (JSM-T300, JEOL, Japan). Pore volume and distribution of pore diameter 
were measured by mercury intrusion porosimetry (MIP; Carlo Elba, Porosimeter 2000, Italy).

\section{Animal Implant Test}

Thirty-six female Japanese White rabbits, 3 months of age, weighing from 2.3 to $2.4 \mathrm{~kg}$, were used for this study. Animals rearing and experiments were performed at the Biomedical Research Center, Nagasaki Univ., following the Guidelines for Animal Experimentation of Nagasaki Univ. Rabbits were anesthetized before surgery. Under sterile conditions, the distal metaphysis and lateral condyle of the left femur were exposed through a 3-cm long lateral longitudinal skin incision and the thigh muscles were divided. A dead-end defect, $6 \mathrm{~mm}$ in diameter and 10 $\mathrm{mm}$ in depth, was created in the lateral cortex just proximal to the epiphyseal plate using a surgical drill. The orientation of the defect was perpendicular to the sagittal axis of the femur. The hole was irrigated with saline, each test implant was carefully inserted into the hole manually, and the wound was closed layer by layer. Four experimental animals used for each of the HHA and SHA implants were sacrificed by an overdose of anesthetic at 2, 4, 12 and 24 weeks after the operation, and two experimental animals for each implant were sacrificed by the same method at 72 weeks after the operation. For fluorescent labeling of the bone tissue, calcein was subcutaneously injected 710 days and 1-3 days before sacrifice at 2, 4, 12, 24 and 72 weeks after the operation.

\section{Radiological and Histological Analyses with Histomorphometry}

The distal portion of the left femur was dissected from the sacrificed animals, and X-ray photographs were taken using a soft X-ray apparatus (TRS-1005, SOFRON, Japan). All the harvested tissue specimens were fixed in $4 \%$ formaldehyde in $0.1 \mathrm{M}$ phosphate buffer (pH 7.2), embedded in 2-hydroxyethyl methacrylate/methyl methacrylate/2-hydroxyethyl acrylate mixed resin, and sectioned $3 \mu \mathrm{m}$ thick [8]. These sections were stained with toluidine blue or histochemically stained for tartrate-resistant acid phosphatase (TRAP) activity as described previously [9]. Histomorphometric analyses were performed using the Osteoplan II system (Carl Zeiss, Thornwood, NY) as described previously [10].

\section{RESULT AND DISCUSSIONS}

\section{Character of Samples}

According to $\mathrm{XRD}$, no phases other than HA was detected for HHA and SHA. These results showed that both HHA and SHA were pure HA. When HHA was heated at $900{ }^{\circ} \mathrm{C}$, decomposition of HA to $\beta$-TCP was detected because the HHA was nonstoichiometric HA of calcium-deficient composition. There was no decomposition as a result of heating in the case of SHA because SHA was stoichiometric. The results corresponded to chemical analysis by ICP-MS. HHA was composed of rod-shaped particles about $20 \mu \mathrm{m}$ in length, which were tangled together to form small pores about $0.2 \mu \mathrm{m}$ in size. These crystals were elongated along the $c$-axis. SHA was composed of globular particles, which fused to one another and formed small pores about $0.5 \mu \mathrm{m}$ in size. The total porosity of HHA and SHA was $70 \%$.

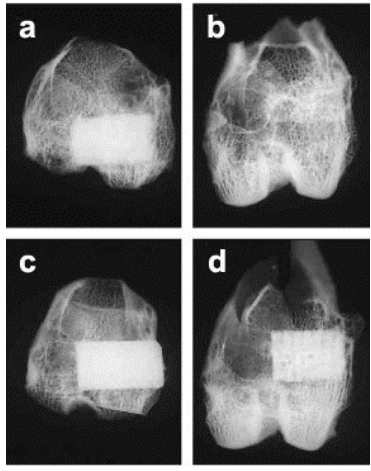

FIGURE 1. Radiographs of rabbit femurs after the operation at 24 weeks (a: HHA, c: SHA) and 72 weeks (b: HHA, d: SHA).

\section{Radiological and Histological Analyses}

Radiographs of rabbit femurs 24 and 72 weeks after the operation were shown in FIGURE 1. At 4 weeks post-surgery, the implanted HHA was recognized clearly by its amorphous radiopacity on Xray film, and there was no difference from the finding of SHA. At 12 weeks, radiopacity derived from HHA was evident, but the margin was a little unclear. At 24 weeks, no difference was seen compared to the radiopacity at 12 weeks post-surgery. The radiographic appearance of implanted SHA was similar from 2 to 24 weeks after the operation. After 72 weeks, radiopacity of SHA was similar to those in earlier stages.

After 2 weeks, TRAP-positive osteoclasts were seen on the surface of HHA. Part of the implant directly contacted with bone. After 4 weeks on HHA, the histological features were similar to those at 2 weeks. Bone tissues in contact with HHA and TRAPpositive cells in contact with HHA were seen. After 12 weeks, the invasion of newly formed bone tissue into HHA implants was evident and many osteoclasts in 
contact with newly formed bone or HHA were seen. After 24 weeks, a considerable amount of HHA remained, however, the amount of newly formed bone invading the implant had increased compared with at 12 weeks. Infiltration of TRAP-positive osteoclasts was still evident. No evidence of bioresorption of the SHA implant was seen from 2 to 24 weeks after the operation. TRAP-positive osteoclasts were seen on the surface of the implant from 2 to 24 weeks after the operation, but there were fewer than those in contact with HHA. After 72 weeks, most HHA was resorbed and substituted with newly formed bone tissue. No evidence of osteoclastic resorption was seen on SHA.

\section{Histomorphometry}

After 24 weeks, Bone volume/tissue volume (BV/TV) in specimens implanted with HHA was significantly greater than that of SHA (TABLE 1). The fluorescent signal derived from calcein labeling was analyzed in the specimens 12 and 24 weeks after the implantation of either HHA or SHA. The width of double labeling was greater in specimens implanted with HHA than in those implanted with SHA at 12 weeks post-operation, but was not clear at 24 weeks. Mineral apposition rate (MAR) and bone formation rate/bone surface (BFR/BS) for newly formed bone tissue in each sample were quantified by histomorphometry. MAR was significantly increased in specimens implanted with HHA in comparison to specimens implanted with SHA at 12 and 24 weeks post-operation. BFR/BS revealed a strong tendency to increase in specimens implanted with $\mathrm{HHA}$ as compared with SHA at 12 and 24 weeks after the operation. Additionally, number of osteoclasts/bone perimeter (N.Oc/B.Pm) and osteoclast surface/bone surface (Oc.S/BS) were quantified and both were significantly increased in specimens implanted with HHA compared with those implanted with SHA at 12 and 24 weeks after the operation.

TABLE 1. Histomorphometry at 24 weeks after implantations.

\begin{tabular}{cccccc}
\hline & BV/TV** & MAR* & BFR/BS & N.Oc/B.Pm* & Oc.S/BS* \\
\hline & $\%$ & $\mu \mathrm{m} / \mathrm{d}$ & $\mu \mathrm{m}^{3} / \mu \mathrm{m}^{2} / \mathrm{d}$ & per $100 \mathrm{~mm}$ & $\%$ \\
\hline SHA & $22.0 \pm 0.2$ & $1.01 \pm 0.09$ & $0.14 \pm 0.05$ & $30.0 \pm 22.5$ & $0.9 \pm 0.5$ \\
HHA & $41.5 \pm 5.0$ & $1.20 \pm 0.07$ & $0.25 \pm 0.09$ & $121.0 \pm 49.0$ & $4.8 \pm 2.2$ \\
\hline \multicolumn{5}{c}{$* \mathrm{P}<0.05, * * \mathrm{P}<0.01$}
\end{tabular}

\section{Discussions}

In resorption by osteoclast and phagocytosis, lysosomal enzymes which have optimum $\mathrm{pH}$ in acidic would play an important role, and lysosome would maintain an acidic internal environment by lysosomal proton pump. The solubility of HA is important to consider the behavior of $\mathrm{HA}$ in vivo. In the past reports, the soluble $\mathrm{pH}$ of $\mathrm{HA}$ in the simulated physiological solution was shown (TABLE 2). It was estimated the $\mathrm{pH}$ of body fluid saturated for HA by making a comparison between solubility curve of HA in the system $\mathrm{Ca}(\mathrm{OH})_{2}-\mathrm{H}_{3} \mathrm{PO}_{4}-\mathrm{H}_{2} \mathrm{O}$ [11-13]. The $\mathrm{pH}$ value was made a difference due to preparation condition for material and experimental temperature. For example, the higher solubility product obtained for HA heated in air, relative to the product obtained for the sample heated in steam, is most probably due to structural changes induced by the thermal treatment [11]. This consideration was revealed by UV Raman in situ measurement for $\mathrm{HA}$ at temperatures up to $1200{ }^{\circ} \mathrm{C}$ reported previously [14-16]. McDowell $\mathrm{H}$. et al. [12]. reported that the solubility of HA decreased with increasing temperature at range of $5^{\circ} \mathrm{C}$ to $37^{\circ} \mathrm{C}$.

TABLE 2. The estimated soluble $\mathrm{pH}$ of $\mathrm{HA}$ in past reports [11-13].

\begin{tabular}{lcl}
\hline \multicolumn{1}{c}{ Preference } & Temperature $/{ }^{\circ} \mathrm{C}$ & $\mathrm{pH}$ value (Comment) \\
\hline $\begin{array}{l}\text { Moreno E.C. et al. } \\
\text { (1968) }\end{array}$ & 25 & 5.6 (heated in air) \\
& 25 & 5.1 (heated in steam) \\
$\begin{array}{l}\text { Mcdowell H. et al. } \\
\text { (1977) }\end{array}$ & 5 & 5.3 (wet process) \\
& 15 & 5.1 (wet process) \\
& 25 & 5.0 (wet process) \\
& 37 & 4.8 (wet process) \\
$\begin{array}{l}\text { Verbeek R.M.H. et al. } \\
\text { (1980) }\end{array}$ & 25 & 5.0 (wet process) \\
\hline
\end{tabular}

In this study, SHA was non-biodegradable in bone tissue. Throughout the experimental period, TRAPpositive osteoclasts were detected on the surface of SHA, but no evidence of osteoclastic resorption was seen. These data suggested that SHA had the potential to be recognized, but was unable to be resorbed by osteoclasts in bone. We previously showed that calcium concentration did not increase at all in the culture media of SHA, in which many osteoclasts were induced by RANKL-expressing cells [8]. Previous data also suggested that osteoclasts could not resorb SHA. We showed that HHA had been well-recognized by osteoclasts for a long period after implantation, and mild biodegradability and good substitution for newly formed bone were detected in animal experiments. Previously, calcium-deficient HA was introduced in biological analyses [17], but the purity and uniformity of the material were uncertain. Biodegradation of HHA by osteoclasts during the experimental period showed that molar ratio of $\mathrm{Ca} / \mathrm{P}$ directly affected osteoclast activity, even though the organic components in bone tissue might also affect osteoclast activity. Biodegradation of HHA was very slow, but 
most part of HHA was replaced by newly formed bone up to 72 weeks after implantation. These data suggested that the solubility of bone substitute did not always correlated to replaceability by bone tissue. We hypothesized that if a bone substitute was continuously recognized and resorbed by osteoclasts, continuous resorption would lead to replacement by new bone tissue irrespective of the mild solubility. Osteoblast activity in the implanted region of HHA was suggested to be higher than that of SHA from histomorphometric data, which showed that MAR was significantly higher in HHA than in SHA at 12 and 24 weeks after implantation. Bone formation is thought to be coupled with bone resorption in that osteoblasts express one of the key molecules for osteoclastogenesis, RANKL [18]. Conversely, the biological mechanism to stimulate osteoblasts by osteoclasts remains uncertain, but the biodegradable nature of HHA was suggested to be associated with the excess bone formation found in animals implanted with HHA. Previously, we have shown that the microstructure of $\beta$-TCP affected the biological response $[8,19]$. It is conceivable that the microstructure of $\mathrm{HA}$ also affects the biological response. HHA was formed by rod-shaped particles just like $\beta$-TCP composed of rod-shaped particles, which was prepared by applied hydrothermal process. $\beta$-TCP composed of rod-shaped particles showed mild and stable degradability compared to conventional $\beta$ TCP composed of globular-shaped particles, and rodshaped particles were suggested to affect the activity of bone cells and metabolism of subsequently formed bone tissue [8]. This might also be the case in HHA. Additionally, HHA is composed of hexagonal crystals with prolonged $c$-axis and the surface of HHA is dominantly a-face. Specific crystal surface of HHA must also influence the biological behavior, because the rod-shaped particles of HHA adsorb negatively charged substances such as albumin selectively [20].

\section{SUMMARY}

Calcium deficient HA prepared hydrothermally was resorbed after implantation at 72 weeks in the bone tissue without addition of any substances and cells such as ES-Cells or iPS-Cells. Hydrothermal method was suitable for preparation of calcium phosphate ceramics for medical application.

\section{ACKNOWLEDGEMENTS}

This research was partially supported by the Ministry of Education, Science, Sports and Culture, Grant-in-Aid for Scientific Research (B), 21300175,
2009. We are greatly indebted to Dr. Atsuo Ito at Advanced Industrial Science and Technology (Japan) and Dr. Hiroyuki Toya at Sumitomo Metal Mining Co. Ltd. (Japan) for their valuable comments.

\section{REFERENCES}

1. Bucholz RW, Carlton A, Holmes R. Clin Orthop Relat Res, 240, 53-62 (1989).

2. Hoogendoorn HA, Renooij W, Akkermans LM, Visser W, Wittebol P. Clin Orthop Relat Res, 187, 281-288 (1984).

3. Ioku K, Kawachi G, Yamasaki N, Toda M, Fujimori H, Goto S. Key Eng Mater, 288-289, 521-524 (2005).

4. Ioku K, Kawachi G, Sasaki S, Fujimori H, Goto S. J Mater Sci, 41, 1341-1344 (2006).

5. Yoshimura M, Suda H, Okamoto K, Ioku K. J Mater Sci 29, 3399-3402 (1994).

6. Ioku K, Nishimura S, Eguchi Y, Goto S. Rev High Pressure Sci Technol, 7, 1398-1400 (1998).

7. Ioku K, Misumi H, Fujimori H, Goto S, Yoshimura M. Proc. 5th Int. Conf. Solvo-thermal Reactions, NJ, USA (2002) pp. 233-236.

8. Okuda T, Ioku K, Yonezawa I, Minagi H, Kawachi G, Gonda Y, Murayama H, Shibata Y, Minami S, Kamihira S, Kurosawa H, Ikeda T. Biomaterials, 28, 2612-21 (2007).

9. Ikeda T, Kasai M, Suzuki J, Kuroyama H, Seki S, Utsuyama M, Hirokawa K. J Biol Chem, 278, 4721747222 (2003)

10. Parfitt AM, Drezner MK, Glorieux FH, Kanis JA, Malluche H, Meunier PJ, et al. J Bone Miner Res, 2, 595-610 (1987).

11. Moreno E.C., Gregory T.M., Brown W.E., J Res Natl Bur Stand-A Phys Chem, 72A, 773-782 (1968).

12. McDowell H., Gregory T.M., Brown W.E., J Res. Natl. Bur. Stand., 81A, 273-281 (1977).

13. Verbeek R.M.H., Steyaer H., Thun H.P., Verbeek F., J C $S$ Faraday I ,76, 209-219 (1980).

14. Toya H., Fujimori H., Ioku K., Goto S., Yoshimura M., Trans MRS-J, 25, 1179-1181 (2000).

15. Ioku K., Fujimori H., Toya H., Goto S., Yoshimura M., "Observation of $\mathrm{OH}$ Ion in Hydroxyapatite by UV Laser Raman Scattering”, Phos. Res. Bull., 11, 13-18 (2000).

16. Fujimori H., Toya H., Ioku K., Goto S., Yoshimura M., Chem. Phys. Lett., 325, 383-388 (2000).

17. Kasten P, Vogel J, Luginbuhl R, Niemeyer P, Tonak M, Lorenz H, Helbig L, Weiss S, Fellenberg J, Leo A, Simank HG, Richter W. Biomaterials, 26, 5879-5889 (2005).

18. Suda T, Takahashi N, Udagawa N, Jimi E, Gillespie MT, Martin TJ. Endocr Rev, 20, 345-357 (1999).

19. Yokozeki H, Hayashi T, Nakagawa T, Kurosawa H, Shibuya K, Ioku K. J Mater Sci Mater Med, 9, 381-384 (1998).

20. Takahashi T, Kamitakahara M, Kawachi G, Ioku K. Key Eng Mater, 361-363, 83-86 (2008). 